\title{
Análisis Panbiogeográfico de Mamíferos del Pleistoceno Tardío y Holoceno
}

\author{
René Josué Monzalvo-López, Jessica Bravo-Cadena, Irene Goyenechea-Mayer G.* \\ Área Académica de Biología, Instituto de Ciencias Básicas e Ingeniería, Universidad Autónoma del Estado de Hidalgo.
}

\begin{abstract}
Resumen
Se realizó un análisis panbiogeográfico para conocer la distribución de Odoicoleus virginianus, Didelphis marsupialis, Cratogeomys merriami y Bison bison del Pleistoceno al Holoceno y comparar sus distribuciones en ambas edades. Se buscaron registros en cuatro bases de datos: GBIF, UNIBIO, REMIB y The Paleobiological database, así como en literatura especializada; los datos fueron analizados en el programa ArcViewGis 3.2 y la extensión "Trazos 2004". Se identificaron los trazos individuales para cada taxón en las dos edades y se obtuvieron los trazos generalizados. Se obtuvo un trazo generalizado para cada edad en donde se observa un cambio significativo en la distribución de $O$. virginianus, $D$. marsupialis y $B$. bison. Los primeros dos tienen una distribución más sureña en el Holoceno mientras que el último amplía su área de distribución hacia el Norte y Sur. En contraste, la distribución de C. merriami se amplía en el Holoceno al centro de México. Los cambios en las distribuciones se relacionan con los cambios climáticos y de vegetación en las dos edades. Cabe destacar que las distribuciones actuales se han dado por el surgimiento de barreras geográficas: Las Montañas Rocosas, Eje Neovolcánico Transversal y la Cordillera Centroamericana.
\end{abstract}

Palabras Clave: Panbiogeografía, mamíferos, Pleistoceno tardío, Holoceno, distribución.

\section{Introducción}

La biogeografía es la disciplina que estudia la distribución de los seres vivos, tanto en el tiempo como en el espacio, considerando también los procesos que dieron lugar a dicha distribución. Esta disciplina presenta dos vertientes: la biogeografía histórica y la biogeografía ecológica (ContrerasMedina et al. 2001).

La biogeografía histórica permite analizar patrones de distribución de especies y taxones supraespecíficos, a escalas espaciales y temporales mayores (Morrone, 2004a). Uno de los métodos de la biogeografía histórica es la panbiogeografía, la cual fue propuesta por León Croizat en 1952. La panbiogeografía es un método de la biogeografía histórica que supone que las barreras geográficas evolucionan junto con las biotas (Morrone, 2000).

El método panbiogeográfico utiliza tres conceptos: Trazo individual, es la unidad básica de un estudio panbiogeográfico, constituye un patrón de interrelación en espacio, tiempo y forma. Se define como las coordenadas primarias de una especie o taxón supraespecífico en el espacio (Morrone, 2004a).

El segundo concepto se refiere a los trazos generalizados: Se reconoce cuando dos o más trazos individuales son compatibles o congruentes (Aguilar-Aguilar y Contreras, 2001). Los trazos generalizados representan biotas ancestrales, ampliamente distribuidas en el pasado en las áreas involucradas, y fragmentadas luego por eventos geotectónicos (Morrone, 2000).

*Autor en correspondencia.

Correo electrónico: ireneg28@gmail.com
Finalmente, se encuentran los nodos, considerados como zonas complejas y compuestas en el sentido geológico y biológico; ocurren cuando dos o más trazos generalizados distintos se intersectan (Aguilar-Aguilar y Contreras, 2001). Éstos fueron interpretados por Croizat como zonas de convergencia geobiótica. Su reconocimiento es uno de los aportes más importantes de la panbiogeografía, y constituye el punto de partida para especular sobre la existencia de áreas compuestas (Morrone, 2004b).

En México se han realizado diversos estudios panbiogeográficos: con mamíferos marinos (Aguilar-Aguilar y Contreras-Medina, 2001), con coleópteros (Márquez y Morrone, 2003) y (Corona y Toledo, 2006), con lepidópteros (Trujano, 2004) y (Salinas-Gutiérrez et al. 2006), con helmintos (Rosas-Valdez y Pérez-Ponce de León, 2004) y (Escalante et al. 2011), con hemípteros (Mariño-Pérez et al. 2007), con himenópteros (Yáñez-Ordóñez et al. 2008) y (Varela-Hernández y Jones, 2013) con peces, helmintos y plantas acuáticas (Martínez-Aquino et al. 2007), con lagartijas (Mejía-Pérez, 2008), con crustáceos (Celis, 2009), con roedores (Barrera-Moreno et al. 2011), con taxones fósiles de plantas acuáticas y terrestres, gasterópodos, ostrácodos, anfibios y mamíferos (Palma-Ramírez et al. 2014) y con plantas (Martínez-Gordillo y Morrone, 2005), (Arana et al. 2011), (Puga-Jiménez et al. 2013), (Quijano-Abril et al. 2014) y (García Díaz et al. 2015).

Para realizar un estudio panbiogeográfico, se necesita de diferentes taxones no relacionados filogenéticamente, para encontrar patrones de distribución congruentes tal y como menciona Morrone (2000) y la aparición de una barrera 
necesariamente afectará a varios organismos a la vez. Es decir, que los patrones de distribución deberían ser repetitivos y no únicos. En el presente trabajo se usó la distribución de cuatro taxones de mamíferos: Odoicoleus virginianus, Didelphis marsupialis, Cratogeomys merriami y Bison bison debido a que muestran un patrón similar de distribución en Norteamérica en el Pleistoceno Tardío y durante el Holoceno, en donde sus distribuciones se amplían y/o reducen a Centroamérica.

Odoicoleus virginianus o venado cola blanca posee una cola característica marrón dorsalmente, blanco ventralmente, y alternando colores lateralmente. Las orejas son aproximadamente el $50 \%$ de la longitud de la cabeza. Las astas de 0 . virginianus tienen una rama principal de la cual surgen verticalmente las ramificaciones. Los adultos tienen una banda blanca en la nariz, la región orbital y el parche de la garganta. Cada lado de la barbilla lleva un punto labial negro. Todas las partes inferiores incluyendo la parte inferior de la cola, el interior de las piernas, el vientre y la barbilla son blancas. Cabe hacer mención que en el hemisferio norte sufren dos mudas completas al año y exhiben variación estacional en el pelaje. La capa de verano, adquirida entre mayo y junio, consiste en pelo corto, delgado y grueso y varía de marrón rojizo a bronce intenso. El pelaje es más oscuro a lo largo del dorso medio y más pálido en la cara, garganta y pecho. La capa de verano se sustituye a finales del verano o principios del otoño por el abrigo de invierno que varía de gris azulado a gris marrón y tiene el pelo más largo, más grueso y más frágil. Los venados cola blanca se extienden desde el sur de Canadá a lo largo de la mayor parte de los Estados Unidos (ausente en Utah, raro en Nevada y California), hasta el norte de Sudamérica. Durante el Pleistoceno, 0 . virginianus se distribuía inicialmente en "América media", la expansión geográfica de $O$. virginianus hacia mayores latitudes fue comparativamente reciente, 0 . virginianus era común en los estados centrales y orientales durante el Irvingtoniano (temprano a mediados de Pleistoceno) (Smith, 1991). De acuerdo con Galindo y Weber (2005) el venado cola blanca es la especie de cérvido con más amplia distribución en México, registrándose en los 32 estados.

Didelphis marsupialis o tlacuache común posee un cuerpo de color negro o gris, con dos capas de pelo: la primera es densa y amarilla o negra, la segunda, o exterior, se caracteriza por ser gruesa, negra o gris. A veces poseen líneas negras en la nariz, que cruzan por los ojos y llegan casi hasta las orejas; por el centro de la corona, desde dentro de las orejas a los ojos; sus mejillas son color amarillo, naranja o blanco opaco; su nariz es rosa; las orejas son puntiagudas y negras; su cola es desnuda, de color negro, y blanco en la punta, y suele ser más larga que la cabeza y el cuerpo juntos, los machos tienden a ser más largos que las hembras; sin embargo, pueden diferir según el país de origen (Aponte, 2013). Son omnívoros y de hábitos mayormente nocturnos (Lozada et al.2015). Se distribuye desde el centro de México hasta el norte de Brasil y Bolivia (IUCN, 2016). Durante el Pleistoceno D. marsupialis se distribuía desde el sureste de los Estados Unidos hasta el centro de México (Paleobiodb.org.2017).

Cratogeomys merriami conocida como tuza de Merriam, tiene orejas y ojos muy pequeños, con cuello poco pronunciado. Cuando excava lo hace con sus fuertes garras y extremidades anteriores, aunque utiliza sus enormes incisivos para desprender rocas o roer raíces que se encuentran en su camino. Sus labios se cierran atrás de los incisivos por lo que no le entra tierra a la boca mientras excava. Las tuzas tapan las entradas a sus madrigueras con tierra, lo cual ayuda a mantener la humedad y la temperatura a un nivel más o menos constante. Vigilan sus túneles para reparar daños y cerrar hendiduras (Ceballos, 2014). C. merriami está restringido al estado de México y el Distrito Federal, así como el norte de Morelos y el centro-oeste de Puebla (Hafner et al. 2005). Durante el Pleistoceno $C$. merriami se distribuía en el centro de México (Paleobiodb.org.2017).

Bison bison o bisonte americano posee pelaje marrón, largo sobre la frente, el cuello, la joroba, y los cuartos delanteros. La cola es relativamente corta, con punta abultada. La cabeza es grande y con cuello corto y grueso. Ambos sexos tienen cuernos cortos y negros que se curvan hacia arriba y hacia adentro, y se estrechan hasta una punta circular, relativamente aguda. Los ojos se colocan antero-lateralmente; las orejas están bien enterradas en el largo pelaje de la cabeza. Las patas son relativamente cortas y fuertes. Los cascos son negros con una impresión casi circular. Los sexos son dimórficos con las hembras similares a los machos pero generalmente presentan un aspecto más pequeño y más ligero El bisonte era extenso en Norteamérica, en Alaska y Canadá occidental a través de los Estados Unidos durante el Pleistoceno; actualmente se distribuye desde el norte de Canadá hasta el Norte de México (Meagher, 1986).

El clima es un factor importante que repercute en la distribución de estos taxones, ya que, durante el Pleistoceno todo el hemisferio norte estuvo afectado por una serie de oscilaciones climáticas externas en la que se alternaron periodos de intenso frio y glaciaciones, con etapas más templadas denominadas interglaciaciones o interestadiales, originadas por causas astronómicas (Álvarez-Lao, 2007). Los estudios paleoclimáticos pueden mostrar las causas de las variaciones climáticas y dan una descripción sobre las características del clima de la Tierra en un determinado momento.

Se sabe que existe un trazo generalizado de México llamado "Norteamericano oriental" que abarca desde el sureste de Canadá hacia la parte del Golfo en México, que finaliza en la parte norte de Centroamérica construido a partir de los trazos individuales de 12 taxones de insectos: Achurum spp., Actias luna-A. truncatipennis, Amblyscirtes celia, Anaea andria, Cyllopsis gemma y Cymatoderella collaris; Angiospermas: Carpinus croliniana, Fagus grandifolia, Liquidambar styraciflua y Nyssa sylvatica y Gimnospermas: Pinus strobus y Taxodium spp. (Contreras-Medina y EliosaLeón, 2001), por lo que se espera que los trazos obtenidos para la distribución de los cuatro taxones estudiados aquí se ubiquen en el trazo generalizado "Norteamericano oriental".

\section{Métodos}

Para la obtención de los registros de las especies del Pleistoceno-Holoceno se tomaron registros provenientes de cuatro bases de datos virtuales: Global Biodiversity Information Facility (GBIF, 2017), Unidad de Informática para la Biodiversidad (UNIBIO, 2017), la Red Mundial de Información Sobre la Biodiversidad (REMIB, 2017) y la base de datos paleontológica (The Paleobiology database, 2017), además de la utilización de artículos especializados (Tabla 1). Para la realización del análisis panbiogeográfico, los datos fueron analizados en el programa ArcViewGis 3.2 
junto con la extensión Trazos 2004, la cual permite unir localidades (mediadas por coordenadas) a través de trazos individuales o generalizados.

Considerando los trazos individuales generados, se realizó una comparación de la vegetación modelada para cada época (Pleistoceno, Holoceno) a partir de los modelos de vegetación de Norte América (CEA, 2017).

Tabla 1. Número de registros correspondiente a cuatro bases de datos virtuales y literatura por taxón.

\begin{tabular}{lllllll}
$\begin{array}{l}\text { Taxones/ } \\
\text { Bases de } \\
\text { datos y } \\
\text { literatura }\end{array}$ & A & B & C & D & $\begin{array}{l}\text { E } \\
1,\end{array}$ & Total \\
\hline $\begin{array}{l}\text { Odoicoleus } \\
\text { virginianus }\end{array}$ & 299 & 14 & 57 & 54 & 5 & 428 \\
\hline $\begin{array}{l}\text { Didelphis } \\
\text { marsupialis }\end{array}$ & 343 & 4 & 22 & 3 & 0 & 372 \\
\hline $\begin{array}{l}\text { Cratogeomys } \\
\text { merriami }\end{array}$ & 22 & 9 & 27 & 0 & 0 & 58 \\
\hline $\begin{array}{l}\text { Bison bison } \\
\text { Total }\end{array}$ & 110 & 0 & 0 & 9 & 0 & 119 \\
\hline A: GB & 27 & 106 & 66 & 5 & 978 \\
\hline
\end{tabular}

A: GBIF, B: UNIBIO, C: REMIB, D: The Paleontology Database, E: Literatura: 1. Gómez-Pérez, L. E., y CarbotChanona, G. (2017); 2. Montellano-Ballesteros, M. M. y Chanona, G. C. (2008); 3. Pérez-Crespo, V. A., ArroyoCabrales, J., y Santos Moreno, A. (2008).

\section{Resultados}

\subsection{Trazos individuales}

Se obtuvieron ocho trazos individuales, cuatro correspondientes al Pleistoceno (Figura 1) y cuatro para el Holoceno (Figura 2), la intersección de los trazos individuales permitió reconocer dos trazos generalizados para cada edad respectivamente.

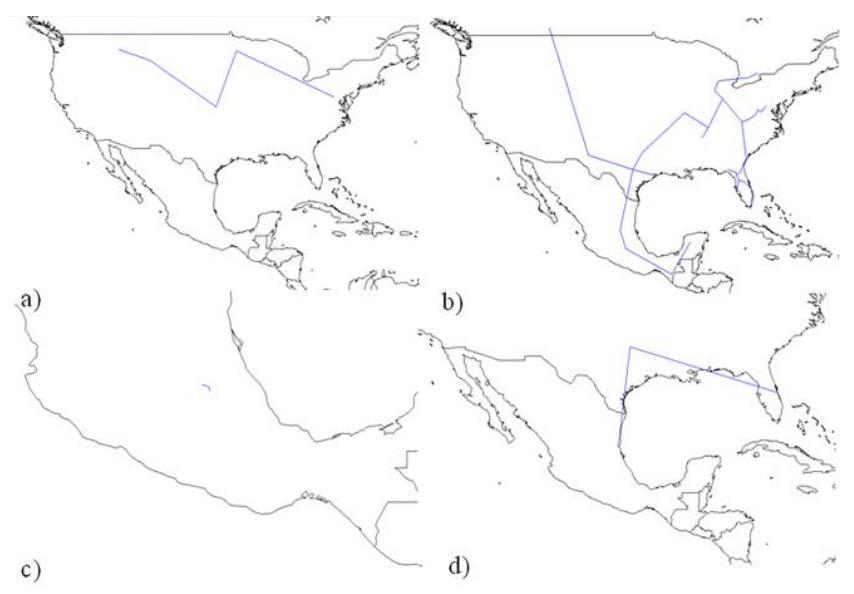

Figura 1. Trazos individuales del Pleistoceno tardío correspondientes a: a) Bison bison, b) Odocoileus virginianus, c) Cratogeomys merriami y d) Didelphis marsupialis.

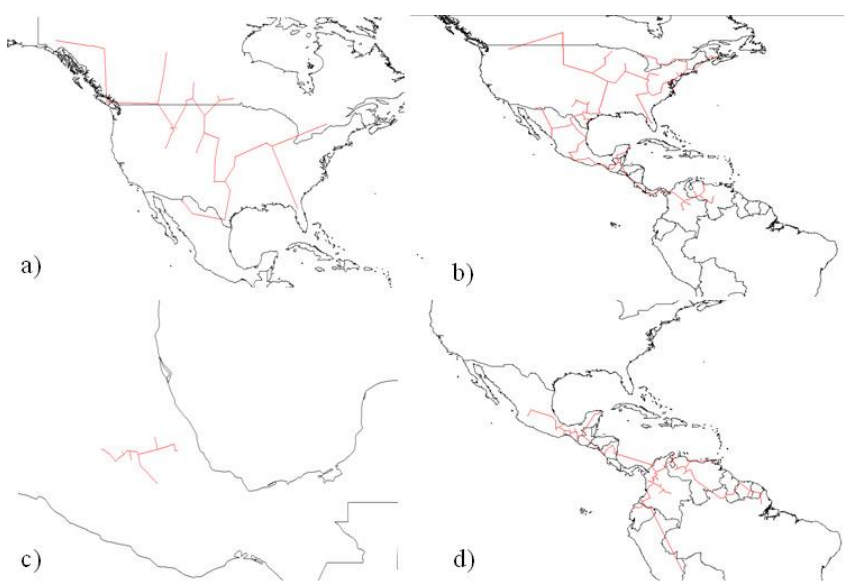

Figura 2. Trazos individuales en el Holoceno de: a) Bison bison, b) Odocoileus virginianus, c) Cratogeomys merriami y d) Didelphis marsupialis.

\subsection{Trazos generalizados}

El trazo generalizado correspondiente al Pleistoceno tardío (Figura 3) abarca la región Noreste, Sur y Noroeste de Estados Unidos. En cuanto al trazo generalizado del Holoceno (Figura 4), se encuentra a partir del Centro de México hasta Centroamérica, específicamente hasta llegar a Venezuela.

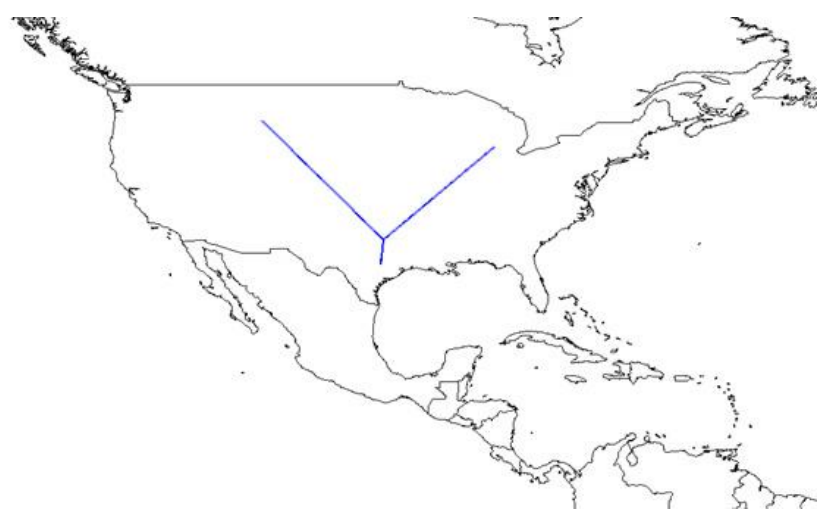

Figura 3. Trazo generalizado del Pleistoceno

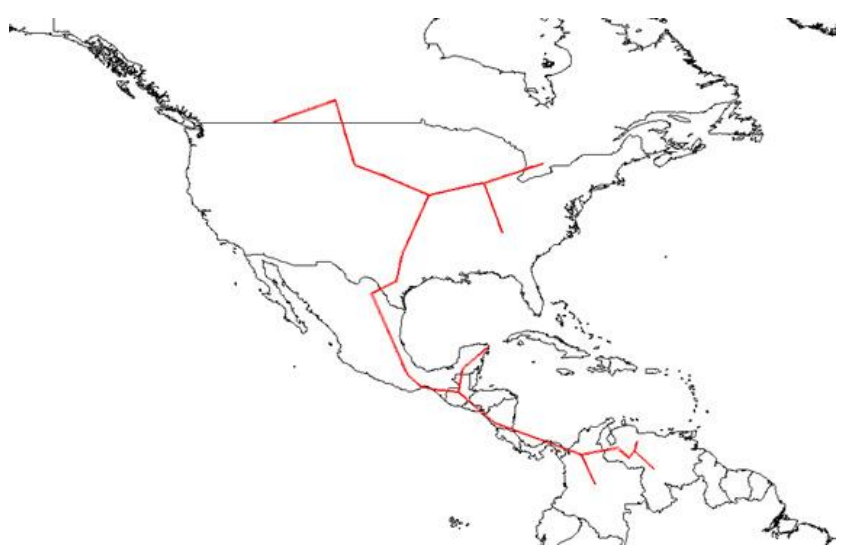

Figura 4. Trazo generalizado del Holoceno. 
Trazo del Pleistoceno tardío. Es un trazo bifurcado que abarca los estados de Montana, Wyoming, Colorado, Oklahoma extendiéndose hacia el sur de Texas por un lado, mientras que la bifurcación se extiende hacia el norte, desde Oklahoma pasando por Missouri y finalizando en el estado de Illinois. Este trazo generalizado se construyó a partir de los trazos individuales de dos taxones (Bison bison y Odocoileus virginianus).

Trazo del Holoceno. Comienza en el estado de Montana hacia la zona sur de Canadá, recorriendo los estados de Dakota del Norte, Dakota del Sur y Iowa. En dicha zona se bifurca: por un lado va hacia el este, pasando por el estado de Wisconsin hasta Michigan, y de ahí se divide de nuevo hacia el sur, pasando por el estado de Illinois. Por el otro lado, comienza desde Iowa y se extiende hacia el sur, cruzando los estados de Kansas, Oklahoma y Texas. Este trazo se extiende hacia el sur en el territorio Mexicano, cruzando por los estados de Coahuila, Tamaulipas, San Luis Potosí, Querétaro, Hidalgo, Puebla, Oaxaca y Chiapas en donde se divide en dos; hacia el este cruzando por Guatemala y Quintana Roo, y hacia el sur cruzando por Guatemala, El Salvador, Nicaragua, Panamá, Colombia y Venezuela. Este trazo generalizado se formó a partir de los trazos individuales de los cuatro taxones $(O$. virginianus, D. marsupialis, C. merriami y B. bison).

\subsection{Comparación de vegetación en el Pleistoceno tardío y Holoceno}

El trazo individual de $B$. bison correspondiente al Pleistoceno Tardío ocurre en vegetación de bosque de taiga de hojas caducifolias, bosque templado de coníferas, matorral tropical xerofítico y arbustos xerófitos templados. En contraste, el trazo individual para el mismo taxón en el Holoceno ocurre en bosque de taiga de hojas caducifolias, bosque mixto fresco, bosque mixto caliente, matorral tropical xerofítico y arbustos xerófitos templados, bosque templado de coníferas, y sabana templada de hoja ancha.

El trazo individual correspondiente a O. virginianus durante el Pleistoceno Tardío se presenta en tundra arbustiva, bosque de taiga de hoja perenne, bosque fresco de coníferas, bosque templado de hoja caduca, bosque templado de coníferas, bosque tropical semidesciduo, matorral tropical xerofítico, arbustos xerófitos templados y bosque mixto caliente. En cuanto a la vegetación correspondiente al trazo individual durante el Holoceno se encuentra en bosque de taiga de hoja perenne, bosque fresco de coníferas, bosque templado de coníferas, matorral tropical xerofítico, bosque de taiga de hoja perenne, bosque tropical semidesciduo, sabana tropical y sabana templada de hoja ancha.

El trazo individual de $C$. merriami durante el Pleistoceno Tardío se encuentra en vegetación de bosque tropical semidesciduo y durante el Holoceno en bosque tropical semidesciduo y bosque mixto caliente.

El trazo individual de D.marsupialis abarca vegetación de bosque tropical semidesciduo, matorral tropical xerofítico, bosque mixto, bosque templado de hoja caduca y bosque templado de coníferas. En cuanto al trazo individual del Holoceno, se encuentra en vegetaciones bosque mixto, bosque tropical siempre verde, bosque tropical semidesciduo, sabana tropical y matorral tropical xerofítico.

\section{Discusión}

Los trazos generalizados indican que una biota ancestral se fue fragmentando por eventos de cambio climático o tectónico (Contreras-Medina y Eliosa-León, 2001). La parte inicial del trazo generalizado del Pleistoceno Tardío se sobrelapa con las Montañas Rocosas, por lo que se puede suponer que éstas fueron la barrera o el evento vicariante que dividió la distribución de los taxones. El trazo generalizado del Holoceno se sobrelapa con los grandes lagos (Michigan, Huron y Erie), así como con la Sierra Madre Oriental en el Norte de México, el Eje Neovolcánico Transversal y con la Cordillera Centroamericana. Este trazo generalizado del Holoceno corresponde con el trazo generalizado "Norteamericano Oriental" y parte del trazo generalizado "Sudamericano" reportado por Contreras-Medina y EliosaLeón (2001).

Con estos resultados se observa que durante las dos épocas existen diferencias en la distribución de los cuatro taxones así como cambios en la vegetación. En general, B. bison cuenta con la misma distribución y se distribuye en los mismos tipos de vegetación en ambas edades, en bosque de taiga de hojas caducifolias, bosque templado de coníferas, matorral tropical xerofítico y arbustos xerófitos templados. Sin embargo, durante el Holoceno se amplió su distribución hacia el noroeste y sur de los Estados Unidos, en tipos de vegetación que incluyen bosque mixto fresco, bosque mixto caliente y sabana templada de hoja ancha.

Los trazos individuales para $O$. virginianus durante el Pleistoceno Tardío y Holoceno comparten las mismas vegetaciones: bosque de taiga de hoja perenne, bosque fresco de coníferas, bosque templado de hoja caduca, bosque templado de coníferas, bosque tropical semidesciduo y matorral tropical xerofítico. Sin embargo, durante el Pleistoceno tardío se distribuía en vegetaciones de arbustos xerófitos templados y bosque mixto caliente, mientras que en el Holoceno se incluyen nuevas vegetaciones que corresponden a bosque de taiga de hoja perenne, sabana tropical y sabana templada de hoja ancha con lo que además se amplía su distribución principalmente hacia zonas sureñas (Centroamérica) y regiones en el noroeste de los Estados Unidos.

Los trazos correspondientes a $C$. merriami durante el Pleistoceno Tardío y Holoceno comparten el tipo de vegetación: bosque tropical semidesciduo; sin embargo, la especie amplió su distribución a bosque caliente mixto en el centro de México.

Los trazos individuales de D. marsupialis en ambas edades comparten los tipos de vegetación: matorral tropical xerofítico y bosque mixto caliente. Durante el Pleistoceno Tardío la distribución de la especie abarcaba regiones de bosque tropical semidesciduo, bosque templado de hoja caduca y bosque templado de coníferas, ubicados en el Sureste de los Estados Unidos. En el Holoceno amplía su distribución hacia el sur de México y Centroamérica encontrándose en vegetaciones de bosque tropical siempre verde, bosque tropical semidesciduo y sabana tropical.

\section{Conclusiones}

Las distribuciones de O.virginianus, D. marsupialis y $B$. bison han tenido un cambio significativo. Durante el Pleistoceno, la distribución de los tres taxones estaba 
principalmente en Norteamérica, mientras que durante el Holoceno O.virginianus D. marsupialis ampliaron su distribución hasta Centroamérica. B. bison amplió su distribución al noroeste y sur de los Estados Unidos. $C$. merriami ha ampliado su distribución en zonas céntricas del país.

Panbiogeographic analysis of late Pleistocene and Holocene mammals.

\begin{abstract}
A panbiogeographic analysis was performed to estimate the distribution of Odoicoleus virginianus, Didelphis marsupialis, Cratogeomys merriami and Bison bison from the Pleistocene to the Holocene and to compare their distributions on both ages. Records from four databases were used: UNIBIO, REMIB, GBIF and The Paleobiological database, as well as specialized literature; data were analyzed with the program ArcViewGis 3.2 and the extension "Trazos 2004". Individual tracks for each taxon were identified for both ages and generalized tracks were obtained. A generalized track was found for each age, showing significant changes in the distribution of $O$. virginianus, D. marsupialis and B. bison. The first two having a southernmost Holocene distribution while the latter extends its range to the North and South. In contrast, the distribution of $C$. merriami expands in the Holocene to the center of Mexico. Changes in distributions are related with climate change and vegetation in both ages. Current distributions respond to the emergence of geographical barriers: the Rocky Mountains, transverse Neovolcanic axis and the Central American Cordillera.
\end{abstract}

Keywords:

Panbiogeography, mammals, late Pleistocene, Holocene, distribution

\section{Agradecimientos}

Al M. en C. Miguel Ángel Cabral Perdomo por la ayuda brindada en los registros del Pleistoceno Tardío.

\section{Referencias}

Aguilar-Aguilar, R., Contreras-Medina, R., 2001. La distribución de los mamíferos marinos de México: un enfoque panbiogeográfico. En: Llorente, J., Morrone, J. J. (Eds.), Introducción a la biogeografía en Latinoamérica: Teorías, conceptos, métodos y aplicaciones. Las Prensas de Ciencias, Facultad de Ciencias, UNAM, México, pp. 197-211.

Álvarez-Lao, D. J., 2007. Revisión paleontológica de los macromamíferos indicadores de clima frío en el Pleistoceno de la Península Ibérica. Tesis Doctoral. Universidad de Oviedo, España.

Aponte, J. D., 2013. Una revisión de la biología del Didelphis marsupialis y su relación con el mal de Chagas y la leishmaniasis. Hipótesis 2013, 96-101.

Arana, M. D., Morrone, J. J., Ponce, M., Oggero, A. J., 2011. Licofitas (Equisetopsida: Lycopodiidae) de las sierras centrales de Argentina: un enfoque panbiogeográfico. Gayana. Botánica 68, 16-21.
Barrera-Moreno, O., Escalante, T., Rodríguez, G., 2011. Panbiogeografía y modelos digitales de elevación: un caso de estudio con roedores en la Faja Volcánica Transmexicana. Revista de Geografía Norte Grande 48, 11-25.

CEA, J., 2017. PMIP 3 Project Home Page. [En linea]: https://pmip3.1sce.ipsl.fr/.

Ceballos, G., 2014. Mammals of Mexico. John Hopkins University Press. Baltimore

Celis, A., 2009. Análisis panbiogeográfico y taxonómico de los cirripedios (Crustacea) de México, Tesis doctoral, Posgrado en Ciencias Biológicas, Universidad Nacional Autónoma de México.

Colecciones de la REMIB., 2017. Conabio.gob.mx. [En línea]: http://www.conabio.gob.mx/remib/doctos/remibnodosd b.html

Contreras-Medina, R., Luna-Vega, I., Morrone, J. J., 2001. Conceptos biogeográficos. Elementos 41, 33-37.

Contreras-Medina, R., Eliosa-León, H., 2001. Una visión panbiogeográfica preliminar de México. En: Llorente, J., Morrone, J. J., (Eds.), Introducción a la biogeografía en Latinoamérica: Teorías, conceptos, métodos y aplicaciones. Las Prensas de Ciencias, Facultad de Ciencias, UNAM, México, pp. 197-211.

Corona, A. M., y Toledo, V. H., 2006. Patrones de distribución de la familia Buprestidae (Coleoptera). En: Morrone, J. J., Llorente-Bousquets, J., (Eds.), Componentes bióticos principales de la entomofauna mexicana, Las Prensas de Ciencias, Facultad de Ciencias, UNAM, México, pp. 333-391.

Escalante, T., Martínez-Salazar, E. A., Falcón-Ordaz, J., Linaje, M., Guerrero, R., 2011. Análisis panbiogeográfico de Vexillata (Nematoda: Ornithostrongylidae) y sus huéspedes (Mammalia: Rodentia). Acta Zoológica Mexicana 27, 25-46.

Free and Open Access to Biodiversity Data | GBIF.org, 2017. Gbif.org. [En línea]: http://www.gbif.org/

Galindo, C., y Weber, M., 2005 Venado cola blanca. En: Los mamíferos silvestres de México En: Ceballos, G., Oliva, G., (Eds.). Comisión Nacional para el Conocimiento y Uso de la Biodiversidad/Fondo de Cultura Económica, México, pp. 517-521.

García Díaz, R., Cuevas Sánchez, J. A., Segura Ledesma, S., y Basurto Peña, F., 2015. Análisis panbiogeográfico de Diospyros spp. (Ebenaceae) en México. Revista Mexicana de Ciencias Agrícolas 6, 187-200.

Gómez-Pérez, L. E., Carbot-Chanona, G., 2017. Contribución al estudio de los megamamíferos del Pleistoceno Tardío del municipio de Villaflores, Chiapas, México. Lacandonia 60, 31-41.

Hafner, M. S., Light, J. E., Hafner, D. J., Brant, S. V., Spradling, T. A., Demastes, J. W., 2005. Cryptic species in the Mexican pocket gopher Cratogeomys merriami. Journal of Mammalogy 86, 1095-1108.

IUCN, International Union for Conservation of Nature, 2016. Didelphis marsupialis. The IUCN Red List of Threatened Species. Version 2017-1. [En línea]: http://maps.iucnredlist.org/map.html?id=40501

Lozada, S., Fernando Ramírez, G., Osorio, H. J., 2015. Características Morfológicas de un Grupo de 
Zarigüeyas (Didelphys marsupialis) del Suroccidente Colombiano. Revista de Investigaciones Veterinarias del Perú 26, 200-205.

Mariño-Pérez, R., Brailovsky, H., Morrone, J. J., 2007. Análisis panbiogeográfico de las especies Mexicanas de Pselliopus Bergroth (Hemiptera: Heteroptera: Reduviidae: Harpactorinae). Acta Zoológica Mexicana 23, 77-88.

Márquez, J., Morrone, J. J., 2003. Análisis panbiogeográfico de las especies de Heterolinus y Homalolinus (Coleoptera: Staphylinidae: Xantholinini). Acta Zoológica Mexicana 90, 15-25.

Martínez Gordillo, M., Morrone, J. J., 2005. Patrones de endemismo y disyunción de los géneros de Euphorbiaceae sensu lato: un análisis panbiogeográfico. Boletín de la Sociedad Botánica de México 77, 21-33.

Meagher, M., 1986. Bison bison. Mammalian species 266 1-8.

Mejía Pérez, J. I., 2008. Análisis panbiogeográfico de los saurios de la Sierra Madre Oriental. Tesis de Licenciatura. Universidad Autónoma del estado de Hidalgo.

Montellano-Ballesteros, M. M., Chanona, G. C., 2008. Presencia de Odocoileus (Artiodactyla, Cervidae) en el Pleistoceno de Chiapas, México. En: Cervantes, F. A., Vargas-Cuenca, J., Hortelano-Moncada, Y., (Eds.), 60 años de la colección nacional de mamíferos del Instituto de Biología, Universidad Nacional Autónoma de México: aportaciones al conocimiento y conservación de los mamíferos mexicanos. Instituto de Biología, UNAM, México.

Morrone, J. J., 2004 a. Homología Biogeográfica: Las Coordenadas Espaciales de la Vida, Vol. 37., $1^{\text {a }}$ Ed. Cuadernos del Instituto de Biología. México: Instituto de Biología, Universidad Nacional Autónoma de México. 199 pp.

Morrone, J. J., 2004 b. Panbiogeografía, componentes bióticos y zonas de transición. Revista Brasileira de Entomología 48, 149.

Morrone, J. J., 2000. Entre el escarnio y el encomio: Léon Croizat y la panbiogeografía. Interciencia 25, 41-47.

Palma-Ramírez, A., Goyenechea, I., Castillo-Cerón, J. M., 2014. Panbiogeografía del área de Santa María Amajac, Hidalgo, México. Revista Mexicana de Biodiversidad 85, 1228-1234.
Pérez-Crespo, V. A., Arroyo-Cabrales, J., Santos Moreno, A., 2008. Generalidades de los mamíferos del Pleistoceno

Tardío de Oaxaca. Naturaleza y Desarrollo 6, 5-11.

Puga-Jiménez, A. L., Andrés-Hernández, A. R., Carrillo-Ruiz, H., Espinosa, D., y Rivas-Arancibia, S. P., 2013. Patrones de distribución del género Zanthoxylum L. (Rutaceae) en México. Revista Mexicana de Biodiversidad 84, 1179-1188.

Quijano-Abril, M. A., Mejía-Franco, F. G., Callejas-Posada, R., 2014. Análisis panbiogeográfico de Enckea (Piperaceae), un pequeño clado de bosques secos en la filogenia de un gran género de bosques húmedos. Revista Mexicana de Biodiversidad 85, 98-107.

Rosas-Valdez, R., y Pérez Ponce de León, G. 2005. Biogeografía histórica de helmintos parásitos de ictalúridos en América del Norte: Una hipótesis preliminar utilizando el método panbiogeográfico. En: Llorente, J., Morrone, J. J., (Eds.), Regionalización biogeográfica en Iberoamérica y tópicos afines: Primeras Jornadas Biogeográficas de la Red Iberoamericana de Biogeografía y Entomología Sistemática (RIBES XII. I-CYTED), Las Prensas de Ciencias, UNAM, México, 217-226 pp.

Salinas-Gutiérrez, J. L., Pozo, C., Luis-Martínez, A., 2006. Relaciones biogeográficas de Papilionidae, Pieridae y Nymphalidae (Rhopalera: Papilionoidea) en el bosque tropical perennifolio de México. Entomotropica 21, 145-152.

Smith, W. P., 1991. Odocoileus virginianus. Mammalian species 388, 1-13.

The Paleobiology Database, 2017. Paleobiodb.org. [En línea]: https://paleobiodb.org/\#/

Trujano, O. M., 2004. Análisis panbiogeográfico de Papilionidae y Pieridae (Lepidoptera: Papilionoidea) en México, Tesis Licenciatura. Facultad de Ciencias, UNAM.

UNIBIO: Unidad de Informática para la Biodiversidad, 2017. Unibio.unam.mx. [En línea]: http://unibio.unam.mx/

Varela-Hernández, F., Jones, R. W., 2013. Patrones biogográficos de hormigas (Hymenoptera: Formicidae) de la Península de Baja California y Sonora, México, mediante el uso de PAE. Dugesiana 20, 111-119.

Yáñez-Ordóñez, O., Trujano Ortega, M., Llorente Bousquets, J., 2008. Patrones de distribución de las especies de la tribu Meliponini (Hymenoptera: Apoidea: Apidae) en México. Interciencia 33,41-45. 